\title{
COMPLETELY CONSERVATIVE DIFFERENCE SCHEMES FOR THE EQUATIONS OF GAS DYNAMICS IN EULER'S VARIABLES*
}

\author{
YU. P. POPOV AND A. A. SAMARSKII \\ Moscow
}

(Received 21 November 1969)

1. THE set of equations of gas dynamics can be written in a variety of ways, each capable of direct physical interpretation. For instance, the energy equation may be written in the divergent form, expressing the variation of the total energy, or in the non-divergent form, expressing the variation of the internal energy only, or in the entropic form etc. These forms are equivalent in the differential form, in the sense that one can be obtained from the other by means of the remaining equations of the set.

When the differential equations are solved numerically by the method of finite differences, it is approximated by a difference scheme. The latter may be based on any one of the equivalent forms of the differential equations. In particular, classical conservative schemes are based on the divergent equations [1]. But the equivalence property does not in general hold for the set of difference equations. For instance, if the non-divergent energy equation is used in the scheme, it cannot always be reduced to the divergent difference form. In the course of transformation, due to "mismatch" of the individual equations of the scheme, remainder terms appear, the presence of which may be treated as due to energy sources of a purely difference type being present in the scheme. These fictitious sources lead to violation of the difference analogue of the law of conservation of total energy.

Similarly, the divergent difference equation cannot be transformed into the corresponding non-divergent form. In this difference scheme, when the law of conservation of total energy is satisfied, the internal energy, and hence the kinetic energy, balance is destroyed.

The energy unbalance depends on the nature of the salution. While it is small on smooth functions, it can become comparable to the total energy on solutions which vary strongly in time and space.

*Zh. vȳchisl. Mat. mat. Fiz. 10, 3, 773-779, 1970. 
In [2], completely conservative difference schemes, which simultaneously approximate all the equivalent sets of differential equations, were devised for the equations of gas dynamics in Lagrangian co-ordinates. In particular, not only are the difference analogues of the basic laws of conservation - of mass, momentum and total energy (as for ordinary conservative schemes) satisfied for these schemes, but also the detailed energy balance, i.e. the balance with respect to the internal and kinetic energy forms individually.

In the present paper we construct conservative difference schemes with the first and second orders of approximation for the equations of gas dynamics in Euler's variables.

2. The set of one-dimensional non-stationary equations of gas dynamics in Eulcr's co-ordinates is

$$
\begin{aligned}
& j=\rho v, \\
& \frac{\partial \rho}{\partial t}=-\frac{\partial j}{\partial r}, \\
& \frac{\partial j}{\partial t}=-\frac{\partial}{\partial r}(p+j v), \\
& \frac{\partial}{\partial t} \rho\left(\varepsilon+0.5 v^{2}\right)=-\frac{\partial}{\partial r} j\left(\varepsilon+0.5 v^{2}\right)-\frac{\partial p v}{\partial r} .
\end{aligned}
$$

Here, $t$ is time, $r$ the space co-ordinate, $j$ the gas flow density, $v$ the velocity, $p$ the pressure, $\rho$ the density, and $\epsilon$ the internal energy of the gas.

After integration with respect to space and time, (2.2) - (2.4) yield respectively the laws of conservation of mass, momentum and total energy for a fixed volume.

The set of equations (2.1) - (2.4) may be written in several equivalent forms. For instance, the equation of motion (2.3) may be transformed with the aid of (2.2) to

$$
\rho \frac{\partial v}{\partial t}+j \frac{\partial v}{\partial r}=-\frac{\partial p}{\partial r}
$$

which expresses the variation of the momentum of a material particle along its trajectory of motion.

Using (2.2) and (2.3), the energy equation (2.4) is equivalent to the following relationships:

$$
\frac{\partial \rho \varepsilon}{\partial t}=-\frac{\partial j \varepsilon}{\partial r}-p \frac{\partial v}{\partial r}
$$




$$
\begin{gathered}
\rho \frac{\partial \varepsilon}{\partial t}+j \frac{\partial \varepsilon}{\partial r}=-p \frac{\partial v}{\partial r} \\
\rho\left(\frac{\partial \varepsilon}{\partial t}+p \frac{\partial}{\partial t}\left(\frac{1}{\rho}\right)\right)+i\left(\frac{\partial \varepsilon}{\partial r}+p \frac{\partial}{\partial r}\left(\frac{1}{\rho}\right)\right)=0
\end{gathered}
$$

which describe the variation of the internal energy only.

We introduce into the part of the space $r, t, r_{0} \leqslant r \leqslant r_{\Lambda}, t \geqslant 0$, the difference mesh $\left\{r_{i}, t^{j}\right\}: r_{i+1}=r_{i}+h_{i}, i=0,1, \ldots, N-1, t^{j+1}=t^{j}+\tau^{j}, j=0,1, \ldots$ To simplify later working, we shall confine ourselves to the case of a uniform mesh $\left(h_{i}=\right.$ $=h=$ const, $\tau^{j}=\tau=$ const). We define on the mesh the velocity and gas flow density mesh functions $v_{i}{ }^{j}$ and $j_{i}{ }^{j}$, with values referred to the base-points $\left(r_{i}, t^{j}\right), i=0,1, \ldots, N$, and the density, pressure and internal energy mesh functions $\rho_{i}{ }^{j}, p_{i}{ }^{j}$ and $\epsilon_{i}{ }^{m}$, with values referred to the half-integer points $\left(r_{i+1 / 2} t^{j}\right)\left(r_{i+1 / 2}=r_{i}+0.5 h, i=0,1, \ldots\right.$, $N-1)$. We shall use the following index-less notation [3] for the mesh functions:

$$
\begin{gathered}
y_{i}^{j}=y, \quad y_{i}^{j+1}=\hat{y}, \quad y^{(\sigma)}=\sigma \hat{y}+(1-\sigma) y, \quad y( \pm 1)=y_{i \pm 1}^{j} \\
\frac{\hat{y}-y}{\tau}=y_{i}, \quad \frac{y(+1)-y}{h}=y_{r}, \quad \frac{y-y(-1)}{h}=y_{-} . \\
\sum_{i=1}^{N-1} y_{i} u_{i} h=(y, u), \quad \sum_{i=0}^{N-1} y_{i} u_{i} h=[y, u), \quad \sum_{i=0}^{N} y_{i} u_{i} h=[y, u] .
\end{gathered}
$$

We have for the difference summation:

$$
\left[y, u_{\S}\right]=-\left[\overline{y_{r}} u\right]+y_{N} u_{N}-y_{-1} u_{0} .
$$

We shall make use of the following expressions for the difference differentiation of a product:

$$
(y u)_{r}=y(+1) u_{r}+u y_{r}, \quad(y u)_{t}=\hat{y} u_{t}+u y_{t}, \quad(u y)_{t}=y^{(0.5)} u_{t}+u^{(0.5)} y_{t},
$$

and the difference identity

$$
y^{\left(\sigma_{2}\right)}=y^{\left(\sigma_{2}\right)}+\left(\sigma_{1}-\sigma_{2}\right) \tau y_{t}
$$

3. We take the following multi-parameter family of difference schemes, approximating the set of equations (2.1) - (2.3), (2.6):

$$
\begin{gathered}
j=\rho v, \\
\rho_{t}=-j_{r}^{\left(\sigma_{1}, \sigma_{2}\right)}, \\
\left.i_{t}=-p_{r}^{\left(\sigma_{s}\right)}-i^{\left(\sigma_{1}, \sigma_{2}\right)}\left(v^{\left(\sigma_{t}\right)}+v^{\left(\sigma_{t}\right)}(-1)\right)\right)_{r}, \\
(\rho \varepsilon)_{t}=-\left(j^{\left.\left(\sigma_{1}, \sigma_{2}\right) \varepsilon^{\left(\sigma_{s}\right)}\right)_{r}-p^{\left(\sigma_{s}\right)} v_{r}^{\left(\sigma_{7}\right)} .}\right.
\end{gathered}
$$

The following notation is used here for writing the derivative with weighting factors:

$$
j^{\left(\sigma_{1}, \sigma_{2}\right)}=\rho^{\left(\sigma_{1}\right)} v^{\left(\sigma_{1}\right)}=\left[\sigma_{1} \hat{\rho}+\left(1-\sigma_{1}\right) \rho\right]\left[\sigma_{2} \hat{v}+\left(t-\sigma_{2}\right) v\right] .
$$

The quantities $0 \leqslant \sigma_{k} \leqslant 1, k=1,2, \ldots, 7$, are the parameters of the scheme, via which 
some form of time interpolation of the terms of the equation is realised.

To ensure a unified representation of the difference equations of the scheme, and of the expressions encountered below, at the boundary points we introduce two fictitious intervals $h_{-1}=0$ and $h_{N}=0$. Then, for instance, $v_{-1}=v_{0}$ in (3.3), and $\epsilon_{N}$ in (3.4) is the value of the internal energy at the mesh boundary base-point $i=N$, etc.

The divergence of the equations (3.2) and (3.3) implies that difference analogues hold for the laws of conservation of mass and momentum for a fixed volume $\left\{r_{0}, r_{N}\right\}$.

The energy equation (3.4) is taken in the non-divergent form. This ensures that the difference internal energy balance is satisfied in the scheme:

$$
\begin{gathered}
{\left.[\rho, \varepsilon)\right|_{j_{1}} ^{j_{2}}+\tau \sum_{j=j_{1}}^{j_{2}}\left\{\left(F_{N}^{j}-E_{0}^{j}\right)-A^{j}\right\}=0,} \\
E_{i}{ }^{j}=j_{i} \underset{\varepsilon_{i}}{\left(\sigma_{1}, \sigma_{2}\right)}, \quad A^{j}=-\left[p^{\left(\sigma_{6}\right)}, v_{r}^{\left(\sigma_{7}\right)}\right) .
\end{gathered}
$$

Relationship (3.5) is obtained by summation over the mesh of Eq. (3.4) for $0 \leqslant i \leqslant$ $\leqslant N-1, t^{j_{1}} \leqslant t^{j} \leqslant t^{i_{2}}$ and expresses the fact that the change in the internal energy of. the gas in a fixed volume $\left\{r_{0}, r_{N}\right\}$ is due to the total work $A$ done by the pressure forces on the gas and the flux of internal energy through the boundaries of the volume $r_{0}$ and $r_{N}$.

Let us find the change in the total energy for the scheme (3.1) - (3.4), For this, we transform the last term in (3.5). In view of (2.12), and recalling (2.14), we have

where

$$
A^{j}=\left[p_{r}^{\left(\sigma_{3}\right)}, v^{\left(\sigma_{2}\right)}\right]-\left(R_{N}^{j}-R_{0}^{j}\right)+D_{1}{ }^{j},
$$

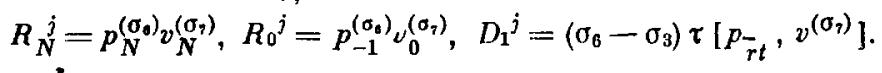

The quantities $p_{-1}$ and $p_{N}$ are the pressures at the boundary base-points of the mesh $i=0$ and $i=. N$.

After multiplying (3.3) by $v^{(07)}$ and summing over the mesh base-points, we get

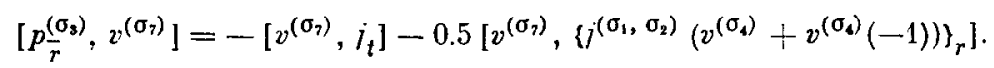

Applying (2.13) for difference differentiation with respect to time and replacing $v^{(\sigma 7)}$ in accordance with $(2.14)$ by $v^{(0.5)}+\left(\sigma_{7}-0.5\right) \tau v_{t}$, we get

$$
\begin{array}{cl}
v^{\left(\sigma_{7}\right)_{t}}=v^{\left(\sigma_{7}\right)} & (\rho v)_{t}=0,5 v^{\left(\sigma_{7}\right)}(\rho v)_{t}+0.5 v^{\left(\sigma_{7}\right)}\left(\rho^{(0.5)} v_{t}+v^{(0.5)} \rho_{t}\right)=0.5\left(\rho v^{2}\right)_{t}+ \\
& +0.5 v^{\left(\sigma_{7}\right)} v^{(0.5)} \rho_{t}+0.5\left(\sigma_{7}-0.5\right) \tau v_{t}\left((\rho v)_{t}+\rho^{(0.5)} v_{t}\right)
\end{array}
$$

We substitute for $\rho_{t}$ in this from the equation of continuity and rewrite (3.7) as 


$$
\begin{gathered}
{\left[p_{r}^{\left(\sigma_{3}\right)}, v^{\left(\sigma_{7}\right)}\right]=-0.5\left[\rho, v^{2}\right]_{t}+0.5\left[v^{\left(\sigma_{7}\right)} v^{(0.5)}, j_{r}^{\left(\sigma_{1}, \sigma_{2}\right)}\right]-} \\
-0.5\left[v^{\left(\sigma_{7}\right)},\left\{i^{\left(\sigma_{1}, \sigma_{2}\right)}\left(v^{\left(\sigma_{4}\right)}+v^{\left(\sigma_{t}\right)}(-1)\right)\right\}_{r}\right]+D_{2}{ }^{j}, \\
D_{2}{ }^{j}=-0.5\left(\sigma_{7}-0.5\right) \tau\left[v_{t},(\rho v)_{t}+\rho^{(0.5)} v_{t}\right] .
\end{gathered}
$$

Further, it can be shown similarly that

$$
\begin{aligned}
& 0.5\left[v^{\left(\sigma_{7}\right)^{(0,5)}} l_{r}^{\left(\sigma_{1}, \sigma_{2}\right)}\right]-0.5\left[v^{\left(\sigma_{7}\right)},\left\{j^{\left(\sigma_{1}, \sigma_{7}\right)}\left(v^{\left(\sigma_{4}\right)}+v^{\left(\sigma_{4}\right)}(-1)\right)\right\}_{r}\right]= \\
& =-\left(W_{N+1}^{j}-W_{0}{ }^{j}\right)+D_{3}{ }^{j}+D_{4}{ }^{j}, \\
& W_{i}{ }^{j}=0.5 j_{i}^{\left(\sigma_{1}, \sigma_{2}\right)} v_{i}^{\left(\sigma_{7}\right)_{v}} v_{i}^{\left(\sigma_{7}\right)}, \quad{ }^{r-1}=v_{J}, \quad v_{N+1}=v_{N} \text {, } \\
& D_{3}^{j}=-0.5\left(\sigma_{4}-0.5\right) \tau\left[v^{\left(\sigma_{7}\right)} i_{l}, l_{r}^{\left(\sigma_{1}, \sigma_{2}\right)}\right] \text {, } \\
& b_{4}{ }^{i}=-11.5\left(\sigma_{4}-\sigma_{7}\right) \tau\left[v^{\left(\sigma_{7}\right)},\left(J^{\left(\sigma_{1}, \sigma_{2}\right)} v_{t}(-1)\right)_{r}+l^{\left(\sigma_{1}, \sigma_{2}\right)}(+1) v_{r t}\right] \text {. }
\end{aligned}
$$

On combining (3.9), (3.8) and (3.6) with (3.5), we get the following difference analogue for the law of conservation of the total energy for the scheme (3.1) - (3.4):

$$
\begin{aligned}
& \left.\{\rho, \rho)\rfloor\}_{\partial_{1}}^{j_{2}}+0 .\left.\bar{u}\left[\rho, v^{2}\right]\right|_{\partial_{1}} ^{j_{3}}+\tau \sum_{j=j_{1}}^{j_{2}}\left(E_{N}^{j}-E_{0}^{j}\right)+\left(R_{N}^{j}-R_{0}^{j}\right)+\left(W_{N+1}^{j}-W_{0}^{j}\right)\right\}=\Delta E, \\
& \Delta E=\tau \sum_{j=j_{1}}^{j_{2}}\left(D_{1}^{j}+D_{2}^{j}+D_{3}^{j}+D_{4}^{j}\right)
\end{aligned}
$$

It is clear that this law is in general violated. The total energy unbalance $\Delta E$ accumulates in time and is of order $O(\tau)$ on smooth solutions. The unbalance $\Delta E$ is independent of the space interval of the mesh and hence cannot be reduced by making the interval $h$ smaller. It is clear from the structure of the terms $D_{k}(k=1.2,3,4)$ that the unbalance depends on the nature of the solution and may reach large values at discontinuities or on solutions that vary strongly in time and space.

To ensure that the difference law of conservation of the total energy ( $\Delta E \equiv 0$, $\left.D_{k} \equiv 0\right)$ is satisfied in our scheme, we only need to satisfy the conditions

$$
\sigma_{6}=\sigma_{3}, \quad \sigma_{7}=\sigma_{4}=0.5 \text {. }
$$

When these conditions are satisfied, the non-divergent energy equation (3.4) is equivalent to the divergent difference equation

$$
(\rho \varepsilon)_{t}+0.5\left(\rho v^{2}\right)_{t}=-\left(j^{\left(\sigma_{1}, \sigma_{2}\right)}\left(\varepsilon^{\left(\sigma_{5}\right)}+0.5 v^{(0.5)} v^{(0.5)}(-1) j\right)_{r}-\left(p^{\left(\sigma_{3}\right)}(-1) v^{(0.5)}\right)_{r} .\right.
$$

This last equation is the difference approximation of the divergent differential equation (2.4). It may be obtained from (3.4) algebraically, by means of (3.1) - (3.3).

In accordance with [2], the formal requirement for the equivalence in the difference form of the non-divergent equation (3.4) to some divergent equation also leads to 
conditions (3.11) and to expression (3.12), and may serve as the basis for selecting completely conservative difference schemes from the family (3.1) - (3.4).

Starting from the equivalence rules, we shall require that (3.4) should also reduce to a difference form, approximating (2.7) and (2.8). We shall quote the results without dwelling on the detailed working.

Equation (3.4) is equivalent to the difference equation

$$
\rho^{(v)} \varepsilon_{t}+f^{\left(\sigma_{1}, \sigma_{2}\right)}(+1) \varepsilon_{r}^{\left(\sigma_{s}\right)}=-p^{\left(\sigma_{s}\right)} v_{r}^{(0.5)}, \quad v=1-\sigma_{3},
$$

with the following values of the parameter $\sigma_{5}$ :

$$
\sigma_{5}=0, \quad \sigma_{5}=0.5, \quad \sigma_{5}=1 .
$$

With the auxiliary condition

$$
\sigma_{2}=0.5, \quad \sigma_{5}=\sigma_{1}
$$

Eq. (3.4) also transforms to

$$
\rho^{(\nu)}\left(\varepsilon_{t}+p^{\left(\sigma_{3}\right)}\left(\frac{1}{\rho}\right)_{t}\right)+i^{\left(\sigma_{1}, 0.5\right)}(+1)\left(\varepsilon_{r}^{\left(\sigma_{1}\right)}+p^{\left(\sigma_{3}\right)}\left(\frac{1}{\rho^{\left(\sigma_{1}\right)}}\right)_{r}\right)=0 .
$$

Equations (3.13) and (3.16) approximate the laws of variation of the internal energy for fixed material particles and ensure that the corresponding difference energy balances are satisfied along their trajectories.

Recall that, in the differential form, the equation of motion (2.3) was reduced by means of the equation of continuity (2.2) to (2.5); in the same way, in the difference form, under conditions (3.11), we can transform (3.3) by using (3.2) to

$$
\rho^{(0.5)} v_{t}=-p_{\frac{r}{r}}^{\left(\sigma_{2}\right)}-0.5\left(j^{\left(\sigma_{1}, \sigma_{3}\right)}(+1) v_{r}^{(0.5)}+i^{\left(\sigma_{1}, \sigma_{2}\right)} v_{\frac{(0.5)}{r}}\right) \text {. }
$$

In short, conditions (3.11), (3.14) and (3.15) distinguish, from the seven-parameter family of difference schemes approximating the set of equations of gas dynamics in Euler's variables, a two-parameter family of schemes which are completely conservative:

$$
\begin{gathered}
j=\rho v \quad\left(j^{(\alpha, 0.5)}=\rho^{(\alpha)} v^{(0.5)}\right), \\
\rho_{t}=-j_{r}^{(\alpha, 0.5)}, \\
j_{t}=p \frac{(\beta)}{r}-0.5\left(j^{(\alpha, 0.5)}\left(v^{(0.5)}+v^{(0.5)}(-1)\right)\right)_{r}, \\
(\rho \varepsilon)_{t}=-\left(j^{(\alpha, 0.5)} \varepsilon^{(\alpha)}\right)_{r}-p^{(\beta)_{r}(0.5)}
\end{gathered}
$$

(the parameter $0 \leqslant \beta \leqslant 1$ is free, while the parameter $\alpha$ can only take one of the three values $0,0.5$, and 1 ).

For the scheme (3.18) - (3.21), the difference laws of conservation of mass, momentum and total energy are satisfied, together with the detailed energy balance, i.e. 
the balance with respect to the individual types of energy, internal and kinetic. In addition, the corresponding energy relationships hold in these schemes along the particle trajectories. The family of schemes (3.18) - (3.21) simultaneously approximates the possible equivalent forms of the set of differential equations of gas dynamics (2.1) - (2.8).

The scheme (3.18) - (3.21) has the order of approximation $0(\tau+h)$. When the parameters $\alpha=\beta=0.5$, the order of approximation is $0\left(\tau^{2}+h\right)$.

4. A family of completely conservative schemes similar to (3.18) - (3.21) may be constructed on a somewhat different pattern:

$$
\begin{gathered}
i=\rho(-1) v \quad\left(j^{(\alpha, 0.5)}=p^{(\alpha)}(-1) v^{(0.5)}\right), \\
\rho_{t}=-l_{r}^{(\alpha, 0.5)}, \\
i_{t}=-p_{\frac{(\beta)}{r}}-0.5\left(j^{(\alpha, 0.5)}(-1)\left(v^{(0.5)}+v^{(0.5)}(-1)\right)\right)_{r}, \\
(\rho \varepsilon)_{t}=-\left(j^{(\alpha, 0.5)} e^{(\alpha)}(-1)\right)_{r}-p^{(\beta)_{r} v_{r}^{(0.5)}} .
\end{gathered}
$$

Here. the mesh function of the gas flow density $j$ is referred as before to the $i$-th mesh base-point, but is defined relative to the density at the left-hand adjacent semiintegral point.

Balance relations similar to those discussed in the previous section hold for the schemes (4.1) - (4.4). By the definition of complete conservatism, this family of schemes also simultaneously approximates the possible equivalent forms of the initial set of differential equations. The equations of motion (4.3) and of energy (4.4) may be transformed algebraically to the corresponding equivalent difference forms. For instance, by analogy with (3.12),

$$
(\rho \varepsilon)_{t}+0.5\left(\rho v^{2}(+1)\right)_{t}=-\left(j^{(\alpha 0.5)}\left(\varepsilon^{(\alpha)}(-1)+0.5 v^{(0.5)} v^{(0.5)}(+1)\right)\right)_{r}-\left(p^{(\beta)} v^{(0.0)}\right)_{r}
$$

A particular consequence of (4.5) is that the kinetic energy for the mesh cell $\left[r_{i}, r_{i+1}\right]$ for the scheme (4.1) - (4.4) is determined from the velocity $v_{i+1}$ at the righthand boundary, as distinct from the previous schemes, where the kinetic energy of a cell is evaluated from the velocity $v_{i}$ at the left-hand boundary.

The order of approximation of the schemes (4.1) $(4.4)$ is $0(\tau+h)$; when $\alpha=\beta=0.5$, the order is $0\left(\tau^{2}+h\right)$.

The difference schemes (3.1) - (3.4) and (4.1) - (4.4) are written on asymmetric patterns and therefore have the first order of space approximation $0(h)$. By using a symmetric pattern. completely conservative schemes can be constructed with the second order of approximation $0\left(h^{2}\right)$. Using ordinary index notation, they are

$$
j_{i}^{j}=0.5\left(\rho_{i+1 / 2}^{j}+\rho_{i-1 / 2}^{i}\right) v_{i}^{j},
$$




$$
\begin{aligned}
& \frac{\rho_{i+1 /,}^{j+1}-\rho_{i+1 / 2}^{j}}{\tau^{j}}=\frac{R i_{+1}^{(\alpha)}\left(v_{i+1}^{j+1}+v_{i+1}^{j}\right)-R_{i}^{(\alpha)}\left(v_{i}^{j+1}+v_{i}^{j}\right)}{2 h_{i}}, \\
& \frac{i_{i}^{j+1}-j_{i}^{j}}{\tau^{j}}=-\left[\beta \frac{p_{i+1 / 2}^{j+1}-p_{i-1 / 2}^{j+1}}{0.5\left(h_{i}+h_{i-1}\right)}+(1-\beta) \frac{p_{i+1 / 2}^{j}-p_{i-1 / 2}^{j}}{0.5\left(h_{i}+h_{i-1}\right)}\right]- \\
& -\left\{\frac{\left[R_{i+1}^{(\alpha)}\left(v_{i+1}^{j+1}+v_{i+1}^{j}\right)+R_{i}^{(\alpha)}\left(v_{i}^{j+1}+v_{i}^{j}\right)\right]\left(v_{i+1}^{j+1}+v_{i+1}^{i}+v_{i}^{j+1}+v_{i}^{j}\right)}{8\left(h_{i}+h_{i-1}\right)}-\right. \\
& \left.-\frac{\left[R_{i}^{(\alpha)}\left(v_{l}^{j+1}+v_{i}^{j}\right)+R_{i-1}^{(\alpha)}\left(v_{i-1}^{j+1}+v_{i-1}^{j}\right)\right]\left(v_{i}^{j+1}+v_{i}^{j}+v_{i-1}^{j+1}+v_{i-1}^{j}\right)}{8\left(h_{i}-h_{i-1}\right)}\right\}, \\
& \frac{\rho_{i+1 / 2}^{j+1} \varepsilon_{i+1 / 2}^{j+1}-p_{i+1 / 2}^{j} \varepsilon_{i+1 / 2}^{j}}{\tau}=-\frac{R_{i+1}^{(\alpha)} E_{i+1}^{(\alpha)}\left(v_{i+1}^{j+1}+v_{i+1}^{j}\right)-R_{i}^{(\alpha)} E_{i}^{(\alpha)}\left(v_{i}^{j+1}+v_{i}^{j}\right)}{2 h_{i}}- \\
& -\left(\beta p_{i+1 / 2}^{j+1}+(1-\beta) p_{i+1 / 2}^{j}\right) \frac{v_{i+1}^{j+1}+v_{i+1}^{j}-\left(v_{i}^{j+1}+v_{i}^{j}\right)}{2 h_{i}},
\end{aligned}
$$

where

$$
\begin{aligned}
& R_{i}^{(\alpha)}=\alpha \frac{\rho_{i+1 / 2}^{j+1}+\rho_{i-1 / 2}^{j+1}}{2}+(1-\alpha) \frac{\rho_{i+1 / 2}^{j}+\rho_{i 1 / 2}^{j}}{2}, \\
& E_{i}^{(\alpha)}=\alpha \frac{\varepsilon_{i+1 / 2}^{j+1}+\varepsilon_{i-1 / 2}^{j+1}}{2}+(1-\alpha) \frac{\varepsilon_{i+1 / 2}^{j}+\varepsilon_{i .1 / \%}^{j}}{2} .
\end{aligned}
$$

The individual equations of this difference scheme are clearly equal to half the sums of the corresponding equations of the asymmetric schemes $(3.1) \cdot(3.4)$ and $(4.1)$ (4.4).

The parameter values $\alpha=\beta=0.5$ distinguish in the scheme (4.6) - (4.9) the only completely conservative difference scheme for the equations of gas dynamics in Euler's variables, with the second order of approximation with respect $t o$ time and space $0\left(\tau^{2}+h^{2}\right)$

If through-computations of possible shock waves are required, a pseudo-viscosity is usually introduced into the difference scheme [4]. All the above results are easily extended to this case: the only modification is to replace $p$ in the equations of motion and energy by the sum of the gas-kinetic pressure and the pseudo-viscosity.

5. The completely conservative difference schemes obtained in this paper give genuine quantitative advantages over other schemes of the same order of approximation, on discontinuous and strongly variable solutions. When evaluating such solutions by means of ordinary schemes, various unbalances arise, the magnitudes of which are particularly large in this case, as may be seen from the structure of the terms $D_{k}^{j}$ evaluated above. To avoid unbalance effects distorting the solution, the time step of the ordinary scheme has to be made too small for practical convenience. 
In the completely conservative schemes, the unbalances are identically zero, this being an algebraic consequence of the initial set of difference equations and unrelated to the sizes of the mesh intervals. The introduction of a completely conservative scheme in fact leads to an increased order of approximation on strongly variable solutions. On smooth solutions, the completely conservative and the ordinary difference schemes yield virtually the same result.

Translated by D. E. Brown

\section{REFERENCES}

1. TIKHONOV, A. N. and SAMARSKII, A. A. On homogeneous difference schemes, Zh. vy Mat. mat. Fiz., 1, 1, 5-63, 1961.

2. POPOV, Yu. P. and SAMARSKII, A. A. Completely conservative difference schemes, Zh. vychisl. Mat. mat. Fiz., 9, 4, 953-958, 1969.

3. SAMARSKII, A. A. A priori estmates for the solution of the difference analogue of a parabolic equation, Zh. vȳchisl. Mat. mat. Fiz., 1, 3, 441-460, 1961.

4. RICHTMEYER, R. D. Difference Methods for Initial-Value Problems. Int. Sci., Wiley, 1967. 